\title{
An Update on Safe Anticoagulation
}

\author{
Reiner K. Mailer ${ }^{1}$ Piotr Kuta ${ }^{1}$ Thomas Renné ${ }^{1,2,3}$ \\ ${ }^{1}$ Institute of Clinical Chemistry and Laboratory Medicine, University \\ Medical Center Hamburg-Eppendorf (UKE), Hamburg, Germany \\ 2 Irish Centre for Vascular Biology, School of Pharmacy and \\ Biomolecular Sciences, Royal College of Surgeons in Ireland, Dublin, \\ Ireland \\ ${ }^{3}$ Center for Thrombosis and Hemostasis (CTH), Johannes Gutenberg \\ University Medical Center, Mainz, Germany
}

\begin{abstract}
Address for correspondence Thomas Renné, MD, PhD, Institute for Clinical Chemistry and Laboratory Medicine, University Medical Center Hamburg-Eppendorf, D-20246 Hamburg, Germany (e-mail: thomas@renne.net).
\end{abstract}

\begin{abstract}
Keywords

- coagulation

- thrombosis

- contact activation

- polyphosphate

- factor XII

Blood coagulation is essential to maintain the integrity of a closed circulatory system (hemostasis), but also contributes to thromboembolic occlusion of vessels (thrombosis). Thrombosis may cause deep vein thrombosis, pulmonary embolism, myocardial infarction, peripheral artery disease, and ischemic stroke, collectively the most common causes of death and disability in the developed world. Treatment for the prevention of thromboembolic diseases using anticoagulants such as heparin, coumarins, thrombin inhibitors, or antiplatelet drugs increase the risk of bleeding and are associated with an increase in potentially life-threatening hemorrhage, partially offsetting the benefits of reduced coagulation. Thus, drug development aiming at novel targets is needed to provide efficient and safe anticoagulation. Within the last decade, experimental and preclinical data have shown that some coagulation mechanisms principally differ in thrombosis and hemostasis. The plasma contact system protein factors XII and XI, high-molecular-weight kininogen, and plasma kallikrein specifically contribute to thrombosis, however, have minor, if any, role in hemostatic coagulation mechanisms. Inherited deficiency in contact system proteins is not associated with increased bleeding in humans and animal models. Therefore, targeting contact system proteins provides the exciting opportunity to interfere specifically with thromboembolic diseases without increasing the bleeding risk. Recent studies that investigated pharmacologic inhibition of contact system proteins have shown that this approach provides efficient and safe thrombo-protection that in contrast to classical anticoagulants is not associated with increased bleeding risk. This review summarizes therapeutic and conceptual developments for selective interference with pathological thrombus formation, while sparing physiologic hemostasis, that enables safe anticoagulation treatment.
\end{abstract}

\section{Classical Anticoagulants}

Thromboembolic diseases have remained the major cause of death and disability in the Western world. Thrombosis is the formation of blood clots that occlude vessels and terminate tissue perfusion leading to ischemia. Thrombosis occurs in both arterial and venous vessels resulting in myocardial infarction, ischemic stroke, and deep-vein thrombosis that eventually results in pulmonary embolism or peripheral vascular disease, respectively. Cumulatively, received

November 4, 2021

accepted after revision

December 8, 2021 (c) 2022. Thieme. All rights reserved. Georg Thieme Verlag KG,

Rüdigerstraße 14,

70469 Stuttgart, Germany 
thromboembolic diseases represent a major medical burden causing around one in four deaths worldwide. The incidence of thrombosis is constantly increasing due to the aging population. Therefore, novel therapeutic strategies for prophylactic and acute thrombosis treatments are needed.

Occlusive clots are formed by activated platelets trapped by fibrin fibers that are produced by the blood coagulation system. Antithrombotic therapies that interfere with pathologic thrombus formation either target platelets or the blood coagulation cascade. Widely used clinical platelet inhibitors include (1) cyclooxygenase (COX) inhibitors, (2) purinergic receptor (P2Y) antagonists, and (3) antagonists of glycoprotein IIb/IIIa (GPIIb/IIIa). ${ }^{1,2}$ In contrast, anticoagulant drugs target single or combinations of coagulation proteins (proteases) thereby reducing thrombin and fibrin formation. The majority of anticoagulants have been known and used in patient care for decades. The polysaccharide heparin is the most commonly used classical anticoagulant drug. Following injection, heparins amplify the activity of the endogenous anticoagulant antithrombin that mainly inhibits active thrombin and factor X (FXa). The anticoagulant activity of heparin unfolds shortly after administration but depends on patients' antithrombin levels. Another class of classical anticoagulants is vitamin $\mathrm{K}$ antagonists (VKA; e.g., phenprocoumon and warfarin). These orally administered drugs prevent the posttranslational modification of coagulation factors II, VII, IX, and X through the blockade of vitamin K-dependent $\gamma$-glutamyl carboxylase, which catalyzes $\gamma$-carboxylation of glutamate residues within Gla-domains which is required for coagulation factor binding to cell surfaces. Defective local assembly (lateral diffusion) of the thrombin-forming enzymes largely impairs the enzymatic turnover and fibrin formation. VKAs are potent anticoagulant drugs and commonly used for prophylaxis and treatment of thrombosis; however, VKAs are indirect and unspecific inhibitors that interfere with both pathologic thrombus formation and physiologic hemostasis. Thus, VKA therapy is associated with a significant bleeding risk and regular monitoring is required to avoid hemorrhagic diathesis due to overdosing. While heparin and VKA target several coagulation factors, hirudin and recombinant/synthetic hirudin derivatives inhibit activated thrombin alone. Independent of their mode of action, classical antithrombotic agents are related with therapy-associated bleeding events. Direct oral anticoagulants (DOACs) selectively target thrombin or FXa. Due to their therapeutic range, DOACs do not require regular monitoring and are therefore predisposed for long-term anticoagulation therapy. Large studies showed that the incidence for hemorrhagic side effects is lower in antithrombotic treatments with DOACs in comparison to VKA; yet, an association with a significant bleeding risk still persists. ${ }^{3}$ Indeed, in the last years, specific antibodies (Idarucizumab) and recombinant modified proteins (Andexanet alfa) have been approved to block DOACs for the treatment of drug-associated hemorrhages. Although antagonizing DOACs in bleeding episodes seems feasible, there is still a significant number of drugassociated bleeding diathesis that can eventually become life-threatening, such as for intracerebral hemorrhage. ${ }^{3}$ Tak- en together, currently used anticoagulants provide thromboprotection, but confer another risk: all currently available anticoagulants increase the risk of bleeding that at least partially offsets the therapeutic benefits of reduced thrombotic risk ( - Table 1 ). Thus, the development of novel and safe anticoagulants for the prevention and treatment of thrombotic diseases is required.

\section{The Factor XII-Driven Contact System}

Factor XII (FXII) is the zymogen form of the plasma serine protease factor XIIa (FXIIa). Zymogen FXII is activated to FXIIa by limited proteolysis and proteolytic cleavage is due to enzymatic activation ("fluid phase activation") or by its unique property to undergo autoactivation following binding ("contact") to negatively charged artificial or biologic surfaces ("contact activation"). FXII surface-induced contact activation provides the mechanistic basis for a commonly used diagnostic coagulation assay, for example, the activated partial thromboplastin time (aPTT) that is used as a clinical parameter to measure the potential of the global plasma coagulation. ${ }^{4,5}$ FXII contact activation induced by white clay materials, celite and kaolin or the phenol antioxidant ellagic acid, is used to test the aPTT, a standard diagnostic coagulation assay that is measured more than half a billion times annually. ${ }^{6}$ On aged erythrocytes, FXII seems to be activated by yet to be identified protease activities. ${ }^{7}$ In blood vessels FXII binds to proteoglycans located on the surface of endothelial cells and this a locally produced FXIla initiates the intrinsic pathway of coagulation via activation of its substrate factor XI (FXI). FXIIa formation also leads to the liberation of the proinflammatory mediator bradykinin (BK) by activating plasma prekallikrein to plasma kallikrein (PKa), which drives the cleavage of high-molecular-weight kininogen (HK) ${ }^{9-11}$ Serpin C1 esterase inhibitor (C1INH) is the major plasma inhibitor of FXIIa and PKa. Deficiency or a dysfunctional C1INH is associated with a BK-mediated lifethreatening inherited swelling disorder, hereditary angioedema (HAE) type I or II, respectively. ${ }^{12,13}$ The proteases FXIIa, PKa, their endogenous inhibitor C1INH, FXIa, and the BK precursor HK together form the plasma contact system. Despite its importance for fibrin formation in clotting assays, FXII had been considered to have no function in vivo and research in this field was dormant for approximately 60 years. Nevertheless, studies on FXII experienced a revival following the discovery of thrombo-protection in FXII-deficient mice. $^{14}$

\section{Novel Concepts in Antithrombotic Therapy}

Ideally, an antithrombotic drug should have strong antithrombotic efficacy both in preventing and treating thrombosis. However, in sharp contrast to all currently used anticoagulants, its antithrombotic activity should not be associated with an increased bleeding tendency. However, in the classical textbook scheme, thrombosis (coagulation in pathologic vascular occlusions) and hemostasis (coagulation at the injury site to terminate bleeding) present the opposite 


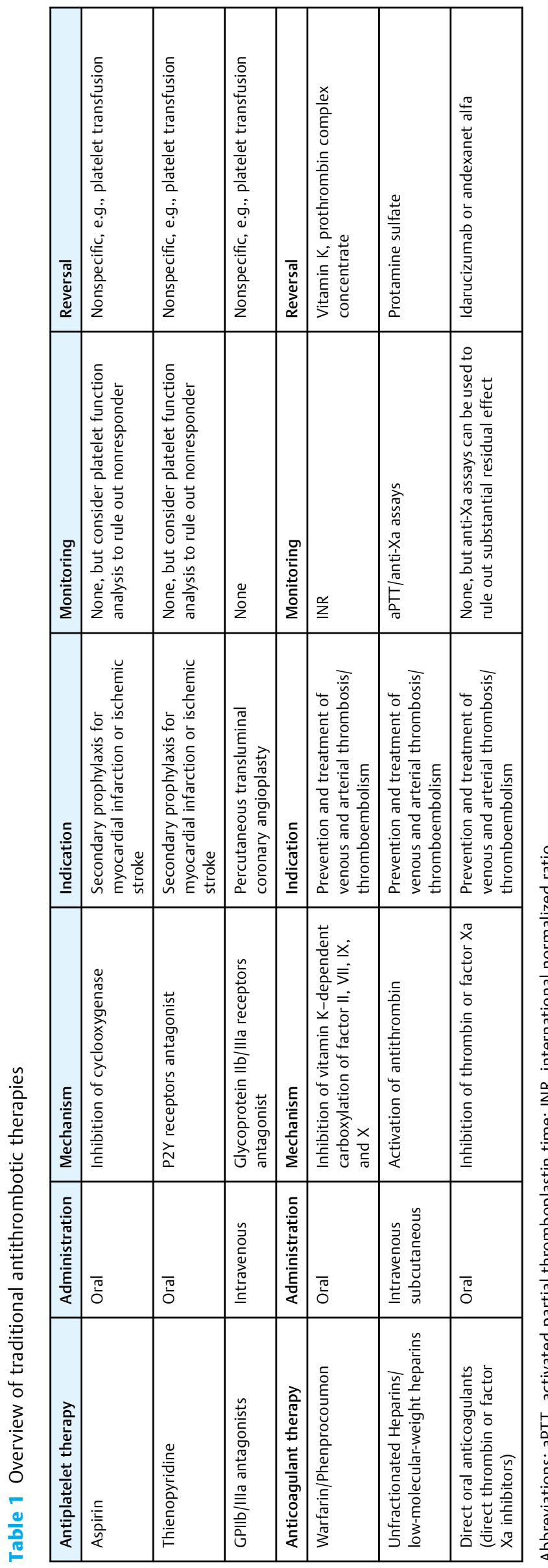

poles of a coagulation balance. Following this fundamental concept, safe anticoagulants, that protect from pathologic thrombus formation, but spare physiologic hemostasis and do not increase bleeding, might not be possible. In 2005, we have originally challenged this concept of the coagulation balance. Our laboratory has generated the first FXII-deficient $\left(\mathrm{F} 12^{-1-}\right)$ mouse model. These mice are protected from occlusive thrombus formation triggered by various chemical and mechanical injury types both in arterial and venous vessels. However, similar to FXII-deficient individuals, the hemostatic potential of $\mathrm{F} 12^{-1-}$ mice was completely normal and exhibited no signs of excessive bleeding at injury sites or defective hemostasis. ${ }^{15}$ Furthermore, reconstitution of $\mathrm{F} 12^{-/}$ - mice with human FXII protein fully restored occlusive thrombus formation, suggesting that FXII could be a target for thrombo-protection that lacks the risk of bleeding. ${ }^{16}$ In addition to experimental vascular injury models, impaired thrombus formation in the absence of FXII has also been shown in atherothrombosis ${ }^{17}$ and ischemic stroke. ${ }^{18}$

\section{Targeting Factor XII in Experimental Thrombosis Models}

The importance of FXII in pathologic thrombosis is not restricted to murine models. We and others have shown a critical role of the FXIIa-driven intrinsic pathway of coagulation for experimental thrombosis in large animals such as rats, ${ }^{19}$ rabbits, ${ }^{20}$ or primates, ${ }^{21}$ and recently in humans. ${ }^{22}$ To address the question which preclinical models have a more predictive value for human therapy, we had screened for antibodies against FXIIa using phage display and demonstrated that recombinant fully humanized antibody 3F7 inhibits FXIIa enzymatic activity, interferes with FXII-mediated coagulation, and blocks experimental thrombosis in mice and rabbits (-Fig. 1). In an adapted extracorporeal membrane oxygenation (ECMO) cardiopulmonary bypass system used for infant therapy, the clinical applicability of 3F7 was tested in rabbits. 3F7 provided thrombo-protection as efficiently as heparin; however, unlike heparin, 3F7 treatment did not impair the hemostatic capacity of animals and did not increase the bleeding risk. Our data provide the first clinically relevant antithrombotic strategy that is not complicated by excess bleeding.

In addition to 3F7, more FXII inhibitors have been developed. rHA-Infestin-4 is a recombinant inhibitor of FXIIa that is composed of the insect protein infestin- 4 that has been fused to human recombinant albumin to increase its half-life and solubility. rHA-Infestin-4 therapy protected from experimental arterial and venous thrombosis and associated thromboembolic diseases such as pulmonary embolism, atherothrombosis, and ischemic strokes. ${ }^{23}$ Similar to 3F7 and despite its potent anticoagulant activities, physiological hemostasis remained unaffected in rHA-infestin-4-treated animals. $^{23}$

Inhibition of FXIIa with rHA-infestin-4 protected mice from $\mathrm{FeCl}_{3}$-induced thrombosis in veins and arteries. ${ }^{24}$ In a model of silent brain ischemia in mice induced by intraarterial injections of microbeads or clot material, rHA- 


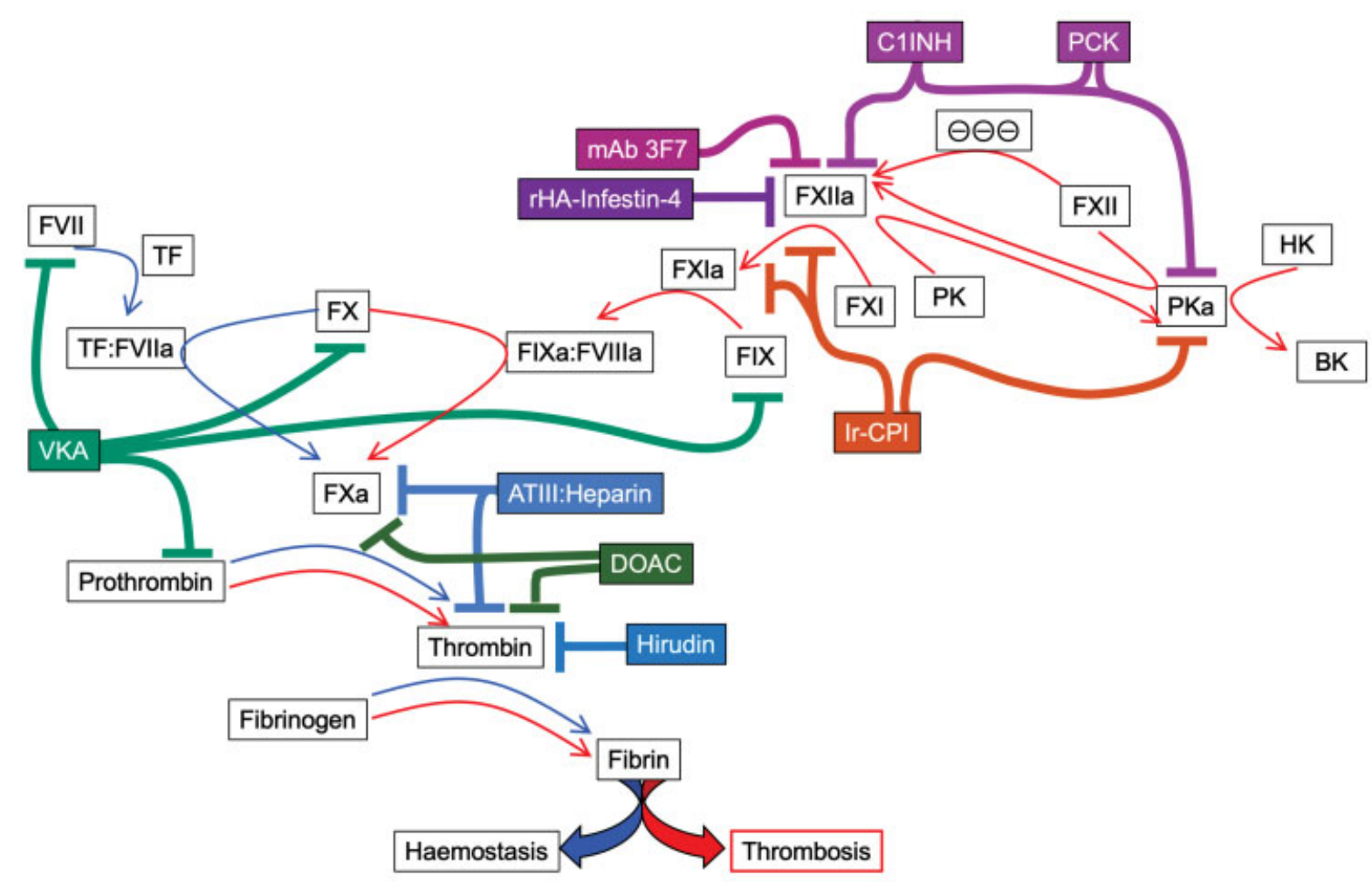

Fig. 1 Regulation of coagulation pathways by antithrombotic agents in hemostasis and thrombosis. Vitamin $\mathrm{K}$ antagonists (VKA) inhibit synthesis of functional coagulation factors VII (FVII), IX (FIX), X (FX), and prothrombin, thereby limiting thrombin generation through the activated complexes tissue factor (TF):FVIla and factor VIIla (FVIIIa):IXa. Heparin potentiates inhibition of FXa and thrombin by binding to antithrombin (AT) III. Direct oral anticoagulants (DOACs) antagonize FXa and thrombin, while hirudin blocks specifically thrombin. Thrombinmediated fibrinogen activation to form insoluble fibrin fibers by the extrinsic coagulation pathway (blue arrows) in hemostasis is exceeded by initiation of the intrinsic coagulation pathway (red arrows). Negatively charged surfaces activate factor XII (FXII) to FXIIa, which is enhanced by a positive feedback loop with FXIla-mediated activation of prekallikrein (PK) to plasma kallikrein (PKa). FXIla and PKa are inhibited by $\mathrm{C} 1$ esterase inhibitor $(\mathrm{C} 1 \mathrm{INH})$ and peptide-based inhibitor (D-Pro-Phe-Arg chloromethyl ketone, PCK). Recombinant Ixodes ricinus inhibitor (Ir-CPI) blocks FXIla and its activated substrate factor XI (FXI) in addition to PKa. Fully humanized monoclonal antibody (mAB 3F7) and infestin-4 from Triatoma infestans fused to recombinant human albumin (rHA-infestin-4) specifically target FXIla and inhibit the FXIla-mediated coagulation cascade.

infestin- 4 also reduced cerebral microinfarcts but was inactive in reducing ischemia-induced inflammatory reactions. ${ }^{25}$ Consistent to the infestin- 4 data, mice treated with Ir-CPI, an inhibitor of FXIIa, activated FXI and PK, were protected from lethal pulmonary embolism and stasis-induced venous thrombosis. ${ }^{26}$ Similarly, FXIIa-driven fibrin formation contributed to stasis-associated vena cava thrombosis induced by restriction of blood flow. ${ }^{27}$ H-D-Pro-Phe-Arg-chloromethyl ketone (PCK), that inhibits FXIla and PK, interfered with lethal pulmonary embolism in murine models. ${ }^{28}$

Consistent with the genetic and pharmacologic models, antisense oligonucleotide (ASO)-mediated "knockdown" of F12 gene expression attenuated experimentally induced arterial and venous thrombosis. ${ }^{29}$ However, in contrast to "classical" anticoagulants, a significant reduction in FXII was required to achieve thrombo-protection. Thrombosis is normal in FXII heterozygous mice ( $122^{+/-}$) having $50 \%$ of normal circulating FXII and FXII needs to be reduced to less than $25 \%$ to provide safe anticoagulant activities. Supporting the key role of the FXII-driven contact system in thrombosis, mice with deficiencies in $\mathrm{PK}^{30,31}$ or $\mathrm{HK}^{32}$ are also protected from thrombosis. More comprehensive overviews about targeting
FXII and contact system proteins in various experimental thrombosis models have been presented in recent reviews. ${ }^{33}$

\section{Factor XI in Thrombosis}

Similar to $\mathrm{F} 12^{-l-}$ mice, animals deficient in its downstream substrate of the intrinsic pathway, coagulation factor XI (FXI), were protected from $\mathrm{FeCl}_{3}$-triggered arterial thrombosis. ${ }^{34}$ However, in contrast to FXII deficiency, low FXI activity is associated with bleeding termed "hemophilia C." Bleeding in FXI deficiency is mild; however, it is rather unpredictable, as it is not linked to circulating FXI antigen levels and biomarkers to assess vascular disease and bleeding risk in these patients are currently lacking. ${ }^{35}$ Despite the striking phenotype in FXI-deficient $\left(\mathrm{F} 11^{-l-}\right)$ mice, the discovery of impaired thrombus formation in these animals did not raise much attention most probably because the underlying mechanism was misinterpreted. Originally it was believed that impaired thrombosis in $\mathrm{F} 11^{-1-}$ mice resulted from defective FXI activation by thrombin that in turn was produced by tissue factor ("feedback activation loop"). ${ }^{36}$ However, later studies challenged this hypothesis. Occlusive 
thrombus formation was similarly defective in $\mathrm{F} 12^{-1-}$ and $\mathrm{F} 11^{-1-}$ mice, as well as in animals with combined deficiency in both clotting factors $\left(\mathrm{F} 12^{-1-} / \mathrm{F} 11^{-1-}\right)$. Similar protection from thrombosis argues against a role of FXI "feedback activation" in thrombotic reactions in vivo but indicates that FXI is mainly activated by FXIIa in pathological platelet-mediated thrombosis. Genetic and pharmacologic targeting of FXI activity has been shown to provide thromboprotection in an array of murine and large animal models and clinical studies on FXI inhibitors in humans are well advanced. In contrast to FXII that triggers thrombo-inflammatory reactions, the role of FXI is restricted to the coagulation system. Thus, targeting FXII provides additional beneficial effects with clinical implications beyond thrombosis.

\section{Targeting Factor XII in Allergy and Inflammation}

In addition to its critical function for thrombosis, the FXIIdriven contact system contributes to allergic disease states. ${ }^{37}$ Upon allergen-challenge mast cells release, the negatively charged polysaccharide heparin that acts as contact activator efficiently activates FXII. Mast cell heparin-driven contact system activation culminates in BK formation that increases vascular permeability through cytoskeleton rearrangements in endothelial cells. ${ }^{38,39}$ FXII contact activation by mast cell heparin has been shown to play a critical role for anaphylactic and allergic diseases both in genetically modified mouse models and patients. Thus, targeting BK generation and signaling provides a novel and alternative treatment strategy for anaphylactic attacks.

Recent findings revealed a role of FXII for SARS-CoV-2associated lung pathology, ${ }^{40}$ suggesting that targeting FXII might help reduce severity and thromboembolic complications in COVID-19 patients. ${ }^{41}$ In contrast to FXII, HK has recently been shown to have a role in experimental liver inflammation. ${ }^{42}$ Together the data reveal differential roles of contact system proteins in distinct inflammatory disease states. $^{43}$

Notably, plasma contact system deficiencies delay the aPTT, demonstrating the dichotomous role of FXIIa for coagulation and inflammation. Patients with deficiencies in functional C1INH develop the rare life-threatening swelling disorder, HAE type I and type II (MIM \#106100). C1INH deficiency aggravates activated mast cell-triggered edema with implications for swelling attacks in HAE. Besides the two classic C1INH-dependent HAE types, a third variant, HAE type III, exists in patients who have completely normal C1INH but similarly suffer from edema attacks. HAE type III (HAE with normal C1INH; FXII-HAE) is associated with missense mutations in the FXII gene resulting in a single point mutation (Thr309Lys or Thr309Arg) that leads to increased FXII enzymatic activity ("gain-of-function"). The underlying mechanism for excessive contact activation remained unknown until it was recently shown that a HAE type III-associated mutant FXII is defective in a mucin-type Thr309-linked glycosylation. Loss of glycosylation led to increased contact-mediated autoactivation of zymogen FXII, resulting in excessive formation of BK. Intravital microscopy imaging revealed that a humanized HAE type III mouse model with inducible liver-specific expression of Thr309Lys-mutated FXII exhibited increased contact-driven microvascular leakage. The FXIIa-neutralizing antibody 3F7 and clinically used PK inhibitors blunted edema in these mice and abolished BK generation in HAE type III patient plasma, suggesting that FXIIa inhibition provides a potential therapeutic strategy to interfere with excessive vascular leakage in HAE and potentially other disease states. ${ }^{44}$

Moreover, application of rHA-infestin- 4 was also protective in mouse models of autoimmune encephalomyelitis ${ }^{45}$ and anaphylactic shock, ${ }^{46}$ illustrating that targeting FXIIa has anti -inflammatory potential in addition to providing antithrombotic protection. Together, the data indicate that FXIla inhibitors have broader clinical applications exceeding their use as antithrombotic drugs.

\section{Polyphosphate: The Natural Factor XII Contact Activator}

The in vivo activation of FXII in the initiating steps of pathologic thrombus formation has been an enigma for many years. ${ }^{4}$ More specific, the natural surface that induces FXII contact activation in vivo had been unknown. ${ }^{47}$ But for decades it has been known that activated platelets induce plasma clotting in a FXII-dependent manner, ${ }^{48}$ indicating that platelets expose FXII-activating agents. Following the discovery that the linear platelet-derived polymer polyphosphate (polyP) serves as the FXII-activating surface, ${ }^{28}$ research elucidated the role of polyP for FXIIa-mediated thrombosis and inflammation ${ }^{5,49}$ (- Fig. 2). Inorganic polyP is a polyanion consisting of up to several hundred phosphates $\left(P_{i}\right)$ linked by energy-rich phosphoanhydride bonds. The chain length of synthetic polyP determines its solubility and FXII activation capacity in plasma, ${ }^{50}$ while natural platelet polyP forms insoluble $\mathrm{Ca}^{2+}$ ion-rich nanoparticles independently of the chain length of the polyP molecule that are retained on the surfaces of procoagulant platelets. ${ }^{51}$ In addition to FXII activation in vivo, polyP has also been reported to modulate several other coagulation reactions in vitro; however, a potential role of these pathways remains to be shown. ${ }^{50}$ Similar to targeting FXIIa, interference with polyP has emerged as a novel strategy for safe inhibition of thrombosis but sparing hemostasis. To interfere with polyP activities, we developed in 2016 the first specific polyP inhibitors, based on recombinant Escherichia coli exopolyphosphatase (PPX) mutants. ${ }^{52}$ Supporting a specific function of polyP in thrombosis, targeting polyP provided thrombo-protection in vivo. Moreover, polyP was visualized as insoluble nanoparticles on the surface of procoagulant platelets in vivo. ${ }^{51}$ The fact that polyPs are insoluble calcium-rich particles that are deposited on activated platelet surfaces explains that procoagulant platelets trigger thrombosis in a FXIIa-dependent manner. In addition to activating FXII, polyP binding to platelet factor 4 (PF4) generates neoantigens that lead to vaccine-induced immune 


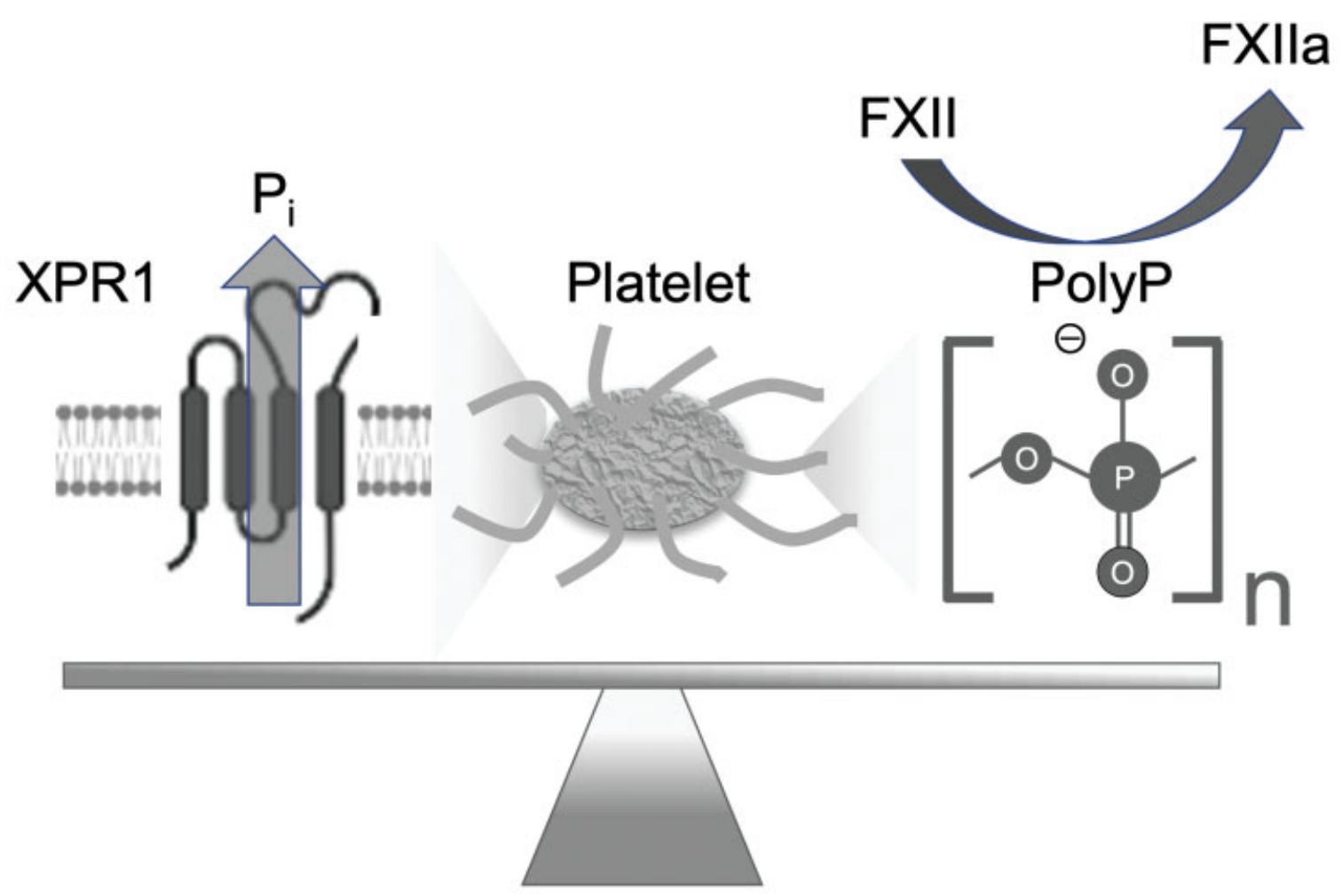

Fig. 2 Regulation of procoagulant platelet polyphosphate. The phosphate exporter Xenotropic and polytropic retrovirus receptor 1 (XPR 1 ) reduces free phosphate $\left(\mathrm{P}_{\mathrm{i}}\right)$ concentration in platelets, which decreases the level of procoagulant polyphosphate (polyP) with implications for factor XII (FXII) contact activation at the membrane of activated platelets.

thrombotic thrombocytopenia (VITT). ${ }^{53}$ Clearly, polyP is a new and exciting player in thrombosis that awaits clinical studies. Indeed, recently a fluorescence-activated cell-sorting (FACS) assay was established to measure polyP in patients using the polyP-binding domain of PPX (PPX_A12) as a polyP probe. ${ }^{52}$ Furthermore, polyP levels can be quantified by chromogenic malachite green assays that measure the formation of phosphate complexes in vitro. Recently, the first regulator for polyP in mammalian systems was identified. The phosphate transporter XPR1 controls polyP in platelets in vivo, and interference with XPR1 modulates thrombosis but not hemostasis both in mouse models and human samples. ${ }^{54}$ Furthermore, the structural basis for polyP-mediated FXII activation that provides the start of polyP-triggered thrombosis and proinflammatory reactions has been elucidated. ${ }^{55} \mathrm{~A}$ specific segment of the FXII C-terminal proline-rich region (termed PR-III) of the heavy chain is required for zymogen contact activation. A FXII variant lacking this sequence can be activated by PKa; however, it is completely defective in undergoing contact activation. The identification of the FXII contact activation side opens perspectives for new coagulation assays. Antibodies directed to the PR-III region induce FXII zymogen "contact" activation fully in solution and in a stoichiometric controllable manner allowing for improved antibody-activated PTT (aaPTT) assays. In contrast to standard aPTT systems, aaPTT assays allow the very precise measurement of FXI levels in the therapeutic range of an emerging target for safe antithrombotic therapy.

\section{Key Points}

- Current anticoagulant drugs reduce risk of thrombosis but concomitantly increase bleeding.

- Genetic and pharmacologic inhibition of contact system factors XII, XI, PK, and HK provides potent thromboprotection without affecting physiological hemostasis.

- Neutralization of factor XII confers additional anti-inflammatory activities.

Conflicts of Interest

The authors declare that they have no conflict of interest.

Acknowledgments

This work was supported in part by grants from the German Research Society (KFO306, SFB877, and SFB841) to T.R.

\section{References}

1 Shevchuk O, Begonja AJ, Gambaryan S, et al. Proteomics: a tool to study platelet function. Int J Mol Sci 2021;22(09): 4776

2 Vara D, Mailer RK, Tarafdar A, et al. NADPH oxidases are required for full platelet activation in vitro and thrombosis in vivo but 
dispensable for plasma coagulation and hemostasis. Arterioscler Thromb Vasc Biol 2021;41(02):683-697

3 Schulman S, Shrum J, Majeed A. Management of bleeding complications in patients with cancer on DOACs. Thromb Res 2016; 140(Suppl 1):S142-S147

4 Long AT, Kenne E, Jung R, Fuchs TA, Renné T Contact system revisited: an interface between inflammation, coagulation, and innate immunity. J Thromb Haemost 2016;14(03): 427-437

5 Nickel KF, Long AT, Fuchs TA, Butler LM, Renné T Factor XII as a therapeutic target in thromboembolic and inflammatory diseases. Arterioscler Thromb Vasc Biol 2017;37(01):13-20

6 Björkqvist J, Nickel KF, Stavrou E, Renné T In vivo activation and functions of the protease factor XII. Thromb Haemost 2014;112 (05):868-875

7 Noubouossie DF, Henderson MW, Mooberry M, et al. Red blood cell microvesicles activate the contact system, leading to factor IX activation via 2 independent pathways. Blood 2020;135(10): 755-765

8 Renné T, Schuh K, Müller-Esterl W. Local bradykinin formation is controlled by glycosaminoglycans. J Immunol 2005;175(05): 3377-3385

9 Hofman Z, de Maat S, Hack CE, Maas C. Bradykinin: inflammatory product of the coagulation system. Clin Rev Allergy Immunol 2016;51(02):152-161

10 Renné T, Gailani D, Meijers JC, Müller-Esterl W. Characterization of the H-kininogen-binding site on factor XI: a comparison of factor XI and plasma prekallikrein. J Biol Chem 2002;277(07): 4892-4899

11 Renné T, Dedio J, Meijers JC, Chung D, Müller-Esterl W. Mapping of the discontinuous H-kininogen binding site of plasma prekallikrein. Evidence for a critical role of apple domain-2. J Biol Chem 1999;274(36):25777-25784

12 Renné T The procoagulant and proinflammatory plasma contact system. Semin Immunopathol 2012;34(01):31-41

13 Björkqvist J, Sala-Cunill A, Renné T Hereditary angioedema: a bradykinin-mediated swelling disorder. Thromb Haemost 2013; 109(03):368-374

14 Renné T, Nieswandt B, Gailani D. The intrinsic pathway of coagulation is essential for thrombus stability in mice. Blood Cells Mol Dis 2006;36(02):148-151

15 Renné T, Pozgajová $M$, Grüner $S$, et al. Defective thrombus formation in mice lacking coagulation factor XII. J Exp Med 2005;202(02):271-281

16 Kenne E, Renné T Factor XII: a drug target for safe interference with thrombosis and inflammation. Drug Discov Today 2014;19 (09):1459-1464

17 Kuijpers MJ, van der Meijden PE, Feijge MA, et al. Factor XII regulates the pathological process of thrombus formation on ruptured plaques. Arterioscler Thromb Vasc Biol 2014;34(08): $1674-1680$

18 Kleinschnitz C, Stoll G, Bendszus M, et al. Targeting coagulation factor XII provides protection from pathological thrombosis in cerebral ischemia without interfering with hemostasis. J Exp Med 2006;203(03):513-518

19 Xu Y, Cai TQ, Castriota G, et al. Factor XIla inhibition by infestin-4: in vitro mode of action and in vivo antithrombotic benefit. Thromb Haemost 2014;111(04):694-704

20 Larsson M, Rayzman V, Nolte MW, et al. A factor XIla inhibitory antibody provides thromboprotection in extracorporeal circulation without increasing bleeding risk. Sci Transl Med 2014;6 (222):222ra17

21 Matafonov A, Leung PY, Gailani AE, et al. Factor XII inhibition reduces thrombus formation in a primate thrombosis model. Blood 2014;123(11):1739-1746

22 Büller HR, Bethune C, Bhanot S, et al; FXI-ASO TKA Investigators. Factor XI antisense oligonucleotide for prevention of venous thrombosis. N Engl J Med 2015;372(03):232-240
23 May F, Krupka J, Fries M, et al. FXIla inhibitor rHA-infestin-4: safe thromboprotection in experimental venous, arterial and foreign surface-induced thrombosis. $\mathrm{Br} \mathrm{J}$ Haematol 2016;173(05): 769-778

24 Hagedorn I, Schmidbauer S, Pleines I, et al. Factor XIla inhibitor recombinant human albumin infestin- 4 abolishes occlusive arterial thrombus formation without affecting bleeding. Circulation 2010;121(13):1510-1517

25 Chen JW, Figueiredo JL, Wojtkiewicz GR, et al. Selective factor XIIa inhibition attenuates silent brain ischemia: application of molecular imaging targeting coagulation pathway. JACC Cardiovasc Imaging 2012;5(11):1127-1138

26 Decrem Y, Rath G, Blasioli V, et al. Ir-CPI, a coagulation contact phase inhibitor from the tick Ixodes ricinus, inhibits thrombus formation without impairing hemostasis. J Exp Med 2009;206 (11):2381-2395

27 von Brühl ML, Stark K, Steinhart A, et al. Monocytes, neutrophils, and platelets cooperate to initiate and propagate venous thrombosis in mice in vivo. J Exp Med 2012;209(04):819-835

28 Müller F, Mutch NJ, Schenk WA, et al. Platelet polyphosphates are proinflammatory and procoagulant mediators in vivo. Cell 2009; 139(06):1143-1156

29 Revenko AS, Gao D, Crosby JR, et al. Selective depletion of plasma prekallikrein or coagulation factor XII inhibits thrombosis in mice without increased risk of bleeding. Blood 2011;118(19): 5302-5311

30 Bird JE, Smith PL, Wang X, et al. Effects of plasma kallikrein deficiency on haemostasis and thrombosis in mice: murine ortholog of the Fletcher trait. Thromb Haemost 2012;107(06): $1141-1150$

31 Göb E, Reymann S, Langhauser F, et al. Blocking of plasma kallikrein ameliorates stroke by reducing thromboinflammation. Ann Neurol 2015;77(05):784-803

32 Merkulov S, Zhang WM, Komar AA, et al. Deletion of murine kininogen gene 1 (mKng1) causes loss of plasma kininogen and delays thrombosis. Blood 2008;111(03):1274-1281

33 Kenne E, Nickel KF, Long AT, et al. Factor XII: a novel target for safe prevention of thrombosis and inflammation. J Intern Med 2015; 278(06):571-585

34 Rosen ED, Gailani D, Castellino FJ. FXI is essential for thrombus formation following $\mathrm{FeCl} 3$-induced injury of the carotid artery in the mouse. Thromb Haemost 2002;87(04):774-776

35 Iglesias MJ, Kruse LD, Sanchez-Rivera L, et al. Identification of endothelial proteins in plasma associated with cardiovascular risk factors. Arterioscler Thromb Vasc Biol 2021;41(12):2990-3004

36 Naito K, Fujikawa K. Activation of human blood coagulation factor XI independent of factor XII. Factor XI is activated by thrombin and factor XIa in the presence of negatively charged surfaces.J Biol Chem 1991;266(12):7353-7358

37 Mailer RK, Rangaswamy C, Konrath S, Emsley J, Renné T An update on factor XII-driven vascular inflammation. Biochim Biophys Acta Mol Cell Res 2022;1869(01):119166

38 Oschatz C, Maas C, Lecher B, et al. Mast cells increase vascular permeability by heparin-initiated bradykinin formation in vivo. Immunity 2011;34(02):258-268

39 Zink J, Frye M, Frömel T, et al. EVL regulates VEGF receptor-2 internalization and signaling in developmental angiogenesis. EMBO Rep 2021;22(02):e48961

40 Englert H, Rangaswamy C, Deppermann C, et al. Defective NET clearance contributes to sustained FXII activation in COVID-19associated pulmonary thrombo-inflammation. EBioMedicine 2021;67:103382

41 Fogarty H, Townsend L, Morrin H, et al; Irish COVID-19 Vasculopathy Study (iCVS) Investigators. Persistent endotheliopathy in the pathogenesis of long COVID syndrome. J Thromb Haemost 2021;19(10):2546-2553

42 Henderson MW, Sparkenbaugh EM, Wang S, et al. Plasminmediated cleavage of high-molecular-weight kininogen 
72 Safe Anticoagulation Mailer et al.

contributes to acetaminophen-induced acute liver failure. Blood 2021;138(03):259-272

43 Rangaswamy C, Mailer RK, Englert H, Konrath S, Renné T The contact system in liver injury. Semin Immunopathol 2021;43(04):507-517

44 Sanrattana W, Smits S, Barendrecht AD, et al. Targeted SERPIN (TaSER): a dual-action antithrombotic agent that targets platelets for SERPIN delivery. J Thromb Haemost 2021. Doi: 10.1111/jth.15554

45 Göbel K, Pankratz S, Asaridou CM, et al. Blood coagulation factor XII drives adaptive immunity during neuroinflammation via CD87-mediated modulation of dendritic cells. Nat Commun 2016;7:11626

46 Sala-Cunill A, Björkqvist J, Senter R, et al. Plasma contact system activation drives anaphylaxis in severe mast cell-mediated allergic reactions. J Allergy Clin Immunol 2015;135(04):1031-1043.e6, e6

47 Rangaswamy C, Englert H, Deppermann C, Renné T Polyanions in coagulation and thrombosis: focus on polyphosphate and neutrophils extracellular traps. Thromb Haemost 2021;121(08):1021-1030

48 Johne J, Blume C, Benz PM, et al. Platelets promote coagulation factor XII-mediated proteolytic cascade systems in plasma. Biol Chem 2006;387(02):173-178
49 Maas C, Renné T Coagulation factor XII in thrombosis and inflammation. Blood 2018;131(17):1903-1909

50 Baker CJ, Smith SA, Morrissey JH. Polyphosphate in thrombosis, hemostasis, and inflammation. Res Pract Thromb Haemost 2018; 3(01):18-25

51 Verhoef JJ, Barendrecht AD, Nickel KF, et al. Polyphosphate nanoparticles on the platelet surface trigger contact system activation. Blood 2017;129(12):1707-1717

52 Labberton L, Kenne E, Long AT, et al. Neutralizing blood-borne polyphosphate in vivo provides safe thromboprotection. Nat Commun 2016;7:12616

53 Greinacher A, Selleng K, Palankar R, et al. Insights in ChAdOx1 nCoV-19 vaccine-induced immune thrombotic thrombocytopenia. Blood 2021;138(22):2256-2268

54 Mailer RK, Allende M, Heestermans M, et al. Xenotropic and polytropic retrovirus receptor 1 regulates procoagulant platelet polyphosphate. Blood 2021;137(10):1392-1405

55 Heestermans M, Naudin C, Mailer RK, et al. Identification of the factor XII contact activation site enables sensitive coagulation diagnostics. Nat Commun 2021;12(01):5596 